Connections between semidefinite relaxations of the Max-Cut and stable set problems

M. Laurent, S. Poljak and F. Rendl

Department of Operations Reasearch, Statistics, and System Theory

BS-R9502 1995 
Report BS-R9502

ISSN 0924-0659

CWI

P.O. Box 94079

1090 GB Amsterdam

The Netherlands

$\mathrm{CWI}$ is the National Research Institute for Mathematics and Computer Science. CWI is part of the Stichting Mathematisch Centrum (SMC), the Dutch foundation for promotion of mathematics and computer science and their applications.

SMC is sponsored by the Netherlands Organization for Scientific Research (NWO). CWI is a member of ERCIM, the European Research Consortium for Informatics and Mathematics.

Copyright @ Stichting Mathematisch Centrum P.O. Box 94079, 1090 GB Amsterdam (NL) Kruislaan 413, 1098 SJ Amsterdam (NL) Telephone +3120 5929333 Telefax +3120 5924199 


\title{
Connections Between Semidefinite Relaxations of the Max-Cut and Stable Set Problems
}

\author{
Monique Laurent ${ }^{1}$ \\ $C W I$ \\ P.O. Box 94079 \\ 1090 GB Amsterdam \\ The Netherlands
}

\author{
Svatopluk Poljak ${ }^{2}$ \\ Fakultät für Mathematik \\ und Informatik \\ Universität Passau \\ Innstr. 33, 94030 Passau \\ Germany
}

\author{
Franz Rendl ${ }^{3}$ \\ Institut für Mathematik \\ Technische Universität Graz \\ Kopernikusgasse 24 \\ A-8010 Graz, Austria
}

\begin{abstract}
We describe the links existing between a recently introduced semidefinite relaxation for the max-cut problem and the well known semidefinite relaxation for the stable set problem underlying the Lovász's theta function. It turns out that the connection between the convex bodies defining the semidefinite relaxations mimicks the connection existing between the corresponding polyhedra. We also show how the semidefinite relaxations can be combined with the classical linear relaxations in order to obtain tighter relaxations.
\end{abstract}

AMS Subject Classification (1991): 90C27, 90C25, 52A20.

Keywords and Phrases: Max-cut problem, stable set problem, semidefinite relaxation.

\section{Introduction}

We consider the following two combinatorial optimization problems: the max-cut problem (3.1) and the maximum stable set problem (3.4). It turns out that, in order to establish the connections existing between these two problems, it is convenient to introduce an intermediate problem, namely, the unconstrained quadratic $(0,1)$-programming problem (3.2), which is well known to be equivalent to the max-cut problem.

\footnotetext{
${ }^{1}$ On leave from LIENS, Ecole Normale Supérieure, Paris.

${ }^{2}$ The research was partly done while the author visited CWI, Amsterdam, with a grant fom the Stieltjes Institute, whose support is gratefully acknowledged. Partially supported also by GACR 0519 .

${ }^{3}$ Research supported by Christian Doppler Laboratorium für Diskrete Optimierung.
} 
A classical approach in the literature to attack these NP-hard problems is the polyhedral approach, leading to the study of the associated polyhedra: the cut polytope (2.1) and the stable set polytope (2.4). A complete description of these polyhedra being out of reach, the general trend was to try to find good linear relaxations of these polyhedra, i.e., relaxations by slightly larger polyhedra over which one could optimize in polynomial time.

Another approach, which has recently received a lot of interest, is to find good nonlinear relaxations of the polyhedra, namely, to find relaxations by (nonnecessarly polyhedral) convex bodies. Such relaxations are generally obtained by requiring positive semidefiniteness of some matrices associated with the problems. Hence, one can optimize over them in polynomial time. This was first done for the stable set problem, when Grötschel, Lovász and Schrijver [GLS88] introduced the convex body $\operatorname{TH}(G)$ as a (in general, nonpolyhedral) relaxation of the stable set polytope. Recently, the elliptope $\mathcal{E}$ was introduced and used in [PR92, LP93, GW94] as a semidefinite relaxation of the cut polytope.

In Section 2, we recall the definitions of the various polytopes and nonpolyhedral sets which are used in connection with the above discrete optimization problems. Formal definitions of the problems are given in Section 3.

Table 1 summarizes the data about the problems and their relaxations. Its columns correspond to the three optimization problems under consideration. The entries in the first row represent the polytopes, defined as the convex hulls of the integer solutions. The second row describes the polytopes obtained as linear relaxations of the problems. These relaxations are derived from the triangle inequalities and the odd cycle inequalities, respectively. Finally the last row corresponds to the semidefinite relaxations of the problems.

A correspondence between the first two columns of Table 1 will be recalled in Section 4.1. It consists of a single mapping $\varphi$ which establishes an isomorphism between the two members in each of the three pairs:

$$
\left(\mathrm{CUT}_{(n+1) \times(n+1)}^{ \pm 1}, \mathrm{BQP}_{n \times n}\right),\left(\mathrm{MET}_{(n+1) \times(n+1)}^{ \pm 1}, \mathrm{BQL}_{n \times n}\right) \text {, and }\left(\mathcal{E}_{n \times n}, \mathcal{Q}_{n \times n}\right) \text {. }
$$

The correspondence between the second and third column of Table 1 is discussed in Section 4.2. It turns out that the entries in the third column are isomorphic to the entries of the second column intersected by a linear subspace. In particular, it is well known that the maximum stable set problem can be formulated in a very simple way as a special case of max-cut problem. Accordingly, it is also well known that the stable set polytope can be formulated as a section of the cut polytope by suitable hyperplanes. We show in addition that their semidefinite relaxations follow the same pattern, i.e., that the body $\operatorname{TH}(G)$ is nothing but a section of the elliptope by the same set of hyperplanes.

The presented connection shows that the maximum stable set problem can be formulated and solved as a constrained max-cut problem, where the constraints 


\begin{tabular}{|c||c|c|c|}
\hline & max-cut & quadr. opt. & stable set \\
\hline \hline integral polytope & $\mathrm{CUT}_{n \times n}^{ \pm 1}$ & $\mathrm{BQP}_{n \times n}$ & $\mathrm{STAB}(\mathrm{G})$ \\
\hline linear relaxation & $\mathrm{MET}_{n \times n}^{ \pm 1}$ & $\mathrm{BQL}_{n \times n}$ & $\mathrm{ODD}(\mathrm{G})$ \\
\hline semidef. relaxation & $\mathcal{E}_{n \times n}$ & $\mathcal{Q}_{n \times n}$ & $\mathrm{TH}(\mathrm{G})$ \\
\hline
\end{tabular}

Table 1: Discrete Optimization Problems and their Relaxations

are linear equations. This is, in fact, true for an arbitrary $(0,1)$-quadratic or linear problem. The max-cut problem captures the underlying $0-1$ structure, and the problem itself can be interpreted as additional constraints to the max-cut problem. (More details are given in Section 6.) In particular, any progress in solving max-cut problem can be potentially utilized for general $(0,1)$-quadratic or linear problems.

Another goal of the paper is to propose a combination of the linear and positive semidefinite relaxations to develope efficient computational schemes. One of the frequently used approaches, the simplex cutting plane algorithm, enables such a combination. For this approach, it is convenient to minimize the number of variables, i.e., to optimize over $\operatorname{MET}^{ \pm 1}(G) \cap \mathcal{E}(G)$ for the max-cut problem. Our results indicate, however, that a tighter approximation can be obtained by optimizing over $\mathrm{MET}_{n \times n}^{ \pm 1} \cap \mathcal{E}_{n \times n}$ (see Section 5). In this approach, the number of variables becomes too large for the simplex algorithm, and an interior point algorithm might be more suitable.

\section{Polyhedra and semidefinite relaxations}

We introduce various geometrical objects - polyhedra, as well as nonpolyhedral convex sets - which are later used in the formulation of combinatorial optimization problems and of their relaxations. Since we work with a larger number of 'objects', we will present them in groups according to the dimension of the underlying space. First, we describe objects lying in the Euclidian space $\Re^{n \times n}$. Then, given a graph $G=(V, E)$ on $n=|V|$ nodes, we introduce some additional convex sets associated with the graph $G$ which are defined in the Euclidean spaces $\Re^{V}, \Re^{E}$ and $\Re^{V+E}$.

\subsection{Convex sets in $\Re^{n \times n}$}

The set of symmetric $n \times n$-matrices is denoted as $\mathrm{SYM}_{n \times n}$. We write $X \succeq 0$ when $X$ is a symmetric positive semidefinite matrix, i.e., if $x^{t} X x \geq 0$ for all $x \in \Re^{n}$. We define the following six convex subsets of $\mathrm{SYM}_{n \times n}$ : $\mathrm{CUT}_{n \times n}^{ \pm 1}, \mathrm{MET}_{n \times n}^{ \pm 1}, \mathcal{E}_{n \times n}$, $\mathrm{BQP}_{n \times n}, \mathrm{BQL}_{n \times n}$, and $\mathcal{Q}_{n \times n}$. 
The cut polytope is defined by

$$
\mathrm{CUT}_{n \times n}^{ \pm 1}:=\operatorname{Conv}\left(x x^{t} \mid x \in\{-1,1\}^{n}\right) .
$$

The metric polytope is defined by

$$
\begin{array}{lll}
\operatorname{MET}_{n \times n}^{ \pm 1}:=\left\{X \in \mathrm{SYM}_{n \times n} \mid\right. & X_{i i}=1 & \text { for } i=1, \ldots, n \\
& X_{i j}-X_{i k}-X_{j k} \geq-1 & \text { for } 1 \leq i, j, k \leq n \\
& X_{i j}+X_{i k}+X_{j k} \geq-1 & \text { for } 1 \leq i, j, k \leq n\}
\end{array}
$$

The inequalities defining $\operatorname{MET}_{n \times n}^{ \pm 1}$ are known as the triangle inequalities. The elliptope $\mathcal{E}_{n \times n}$ is defined by

$$
\mathcal{E}_{n \times n}:=\left\{X \in \mathrm{SYM}_{n \times n} \mid X \succeq 0, X_{i i}=1 \text { for all } i=1, \ldots, n\right\} .
$$

Its members are sometimes called correlation matrices.

The boolean quadric polytope (also called the correlation polytope by some authors) is defined by

$$
\mathrm{BQP}_{n \times n}:=\operatorname{Conv}\left(d d^{t} \mid d \in\{0,1\}^{n}\right) .
$$

The polytope $\mathrm{BQL}_{n \times n}$ consists of the symmetric $n \times n$ matrices $Y=\left(Y_{i j}\right)$ satisfying the inequalities:

$$
\left\{\begin{array}{l}
0 \leq Y_{i j} \leq Y_{i i} \\
Y_{i i}+Y_{j j}-Y_{i j} \leq 1 \\
-Y_{k k}-Y_{i j}+Y_{i k}+Y_{j k} \leq 0 \\
Y_{i i}+Y_{j j}+Y_{k k}-Y_{i j}-Y_{i k}-Y_{j k} \leq 1
\end{array}\right.
$$

for $1 \leq i, j, k \leq n$. The set $\mathcal{Q}_{n \times n}$ is defined by

$$
\mathcal{Q}_{n \times n}:=\left\{Y \in \operatorname{SYM}_{n \times n} \mid Y-\operatorname{diag}(Y)(\operatorname{diag}(Y))^{t} \succeq 0\right\} .
$$

One can check that $\mathcal{Q}_{n \times n}$ is a convex set. (Indeed, let $Y, Y^{\prime} \in \mathcal{Q}_{n \times n}$ with diagonals $d:=\operatorname{diag}(Y), d^{\prime}:=\operatorname{diag}\left(Y^{\prime}\right)$ and let $\alpha$ be a scalar, $0 \leq \alpha \leq 1$. We check that $Y^{\prime \prime}:=\alpha Y+(1-\alpha) Y^{\prime} \in \mathcal{Q}_{n \times n}$; its diagonal is $d^{\prime \prime}:=\alpha d+(1-\alpha) d^{\prime}$. Then, $Y^{\prime \prime}-d^{\prime \prime}\left(d^{\prime \prime}\right)^{t}=\alpha\left(Y-d d^{t}\right)+(1-\alpha)\left(Y^{\prime}-d^{\prime}\left(d^{\prime}\right)^{t}\right)+\alpha(1-\alpha)\left(d-d^{\prime}\right)\left(d-d^{\prime}\right)^{t}$ is indeed positive semidefinite.)

\subsection{Convex sets in $\Re^{V}$}

The stable set polytope of the graph $G=(V, E)$ is defined by

$$
\operatorname{STAB}(G):=\operatorname{Conv}\left(d \in\{0,1\}^{n} \mid d_{i} d_{j}=0 \text { for all edges } i j \in E\right\}
$$


The polytope $\operatorname{ODD}(G)$ in $\Re^{V}$ is defined by the inequalities:

$$
\begin{cases}d_{i} \geq 0 & \text { for } i \in V \\ d_{i}+d_{j} \leq 1 & \text { for } i j \in E \\ \sum_{i \in V(C)} d_{i} \leq \frac{|V(C)|-1}{2} & \text { for each odd cycle } C=(V(C), E(C)) \text { in } G .\end{cases}
$$

The convex body $\operatorname{TH}(G)$ is defined by

$$
\begin{aligned}
& \operatorname{TH}(G):=\left\{d \in \Re^{n} \mid \begin{array}{ll}
\exists z \in \Re^{E\left(K_{n}\right) \backslash E} \text { such that } Z \succeq 0, \\
\text { where } Z \text { is the }(n+1) \times(n+1)
\end{array}\right. \\
& \left\{\begin{array}{lll}
Z_{00}:= & \text { symmetric matrix defined by }(2.7)\} . \\
Z_{0 i}:=Z_{i i}:= & d_{i} & \text { for all } i=1, \ldots, n, \\
Z_{i j}:= & z_{i j} & \text { for all } i j \in E\left(K_{n}\right) \backslash E, \\
Z_{i j}:= & 0 & \text { for all } i j \in E .
\end{array}\right.
\end{aligned}
$$

\subsection{Convex sets in $\Re^{E}$}

We introduce

$$
\operatorname{CUT}^{ \pm 1}(G), \quad \operatorname{MET}^{ \pm 1}(G) \text { and } \mathcal{E}(G)
$$

as the projections of $\mathrm{CUT}_{n \times n}^{ \pm 1}, \mathrm{MET}_{n \times n}^{ \pm 1}$ and $\mathcal{E}_{n \times n}$, respectively, on the subspace $\Re^{E}$ of $\Re^{n \times n}$. The sets $\mathrm{CUT}^{ \pm 1}(G), \mathrm{MET}^{ \pm 1}(G)$ and $\mathcal{E}(G)$ are called the cut polytope, the metric polytope and the elliptope of the graph $G$, respectively.

Note that $\mathrm{CUT}_{n \times n}^{ \pm 1}, \mathrm{MET}_{n \times n}^{ \pm 1}$ and $\mathcal{E}_{n \times n}$ consist of symmetric $n \times n$ matrices with prescribed diagonal entries (namely, equal to 1 ). Such matrices can be encoded by their upper triangular part which is a vector of length $\left(\begin{array}{l}n \\ 2\end{array}\right)$ indexed by the edge set of the complete graph $K_{n}$. Thus

$$
\operatorname{CUT}^{ \pm 1}\left(K_{n}\right), \quad \operatorname{MET}^{ \pm 1}\left(K_{n}\right) \text { and } \mathcal{E}\left(K_{n}\right)
$$

contain the same information as $\mathrm{CUT}_{n \times n}^{ \pm 1}, \mathrm{MET}_{n \times n}^{ \pm 1}$ and $\mathcal{E}_{n \times n}$.

An explicit description of $\operatorname{MET}^{ \pm 1}(G)$ by linear inequalities can be found in [Bar93]. Namely,

$$
\begin{array}{lll}
\operatorname{MET}^{ \pm 1}(G)=\left\{x \in \Re^{E} \mid\right. & -1 \leq x_{e} \leq 1 & \text { for } e \in E \\
& x(F)-x(C \backslash F) \geq 2-|C| & \text { for } F \subseteq C, C \text { cycle },|F| \text { odd }\} .
\end{array}
$$

On the other hand, a parametric description of $\mathcal{E}(G)$ is known for series-parallel graphs [L94].

REMARK 2.8 Note that the vertices of $\operatorname{CUT}^{ \pm 1}(G)$ are the $( \pm 1)$-incidence vectors of the cuts of $G$. It is maybe more customary to represent cuts by their $(0,1)$ incidence vectors, i.e., to consider instead the polytope $\operatorname{CUT}^{01}(G)$ which is in 
one-to-one correspondence with $\mathrm{CUT}^{ \pm 1}(G)$, namely:

$$
\mathrm{CUT}^{01}(G)=\left\{\frac{1-x}{2} \mid x \in \mathrm{CUT}^{ \pm 1}(G)\right\}
$$

(where the vector $\frac{1-x}{2}$ has, by definition, the components $\frac{1-x_{e}}{2}$ for $e \in E$ ).

\subsection{Convex sets in $\Re^{V+E}$}

We introduce

$$
\operatorname{BQP}(G), \quad \operatorname{BQL}(G) \quad \text { and } \quad \mathcal{Q}(G)
$$

as the projections of $\mathrm{BQP}_{n \times n}, \mathrm{BQL}_{n \times n}$ and $\mathcal{Q}_{n \times n}$ on the subspace $\Re^{V+E}$ of $\Re^{n \times n}$ (where $\Re^{V}$ represents the space of the diagonal entries for matrices of $\mathrm{SYM}_{n \times n}$ ).

\section{Combinatorial optimization problems}

We consider the following combinatorial optimization problems: the max-cut problem (3.1), the unconstrained quadratic $(0,1)$-programming problem (3.2), and the maximum stable set problem (3.4).

\subsection{The max-cut problem}

Given a graph $G=(V, E)$ with node set $V:=\{1, \ldots, n\}$ and edge weights $w=$ $\left(w_{e}\right)_{e \in E}$, the max-cut problem can be formulated as

$$
\begin{array}{ll}
\max & \sum_{1 \leq i<j \leq n} \frac{1}{2} w_{i j}\left(1-x_{i} x_{j}\right) \\
\text { s.t. } & x \in\{-1,1\}^{n}
\end{array}
$$

(after setting $w_{i j}=0$ if $i j$ is not an edge of $G$ ). Hence, the cut polytope $\mathrm{CUT}_{n \times n}^{ \pm 1}$ is the convex hull of the set of feasible solutions of the problem (3.1), after linearization of the objective function. In other words, the problem (3.1) can be rewritten as

$$
\begin{array}{ll}
\max & \sum_{1 \leq i<j \leq n} \frac{1}{2} w_{i j}\left(1-X_{i j}\right) \\
\text { s.t. } & X \in \mathrm{CUT}_{n \times n}^{ \pm 1}
\end{array}
$$

Obviously, we have the inclusions:

$$
\mathrm{CUT}_{n \times n}^{ \pm 1} \subseteq \mathrm{MET}_{n \times n}^{ \pm 1} \quad \text { and } \quad \mathrm{CUT}_{n \times n}^{ \pm 1} \subseteq \mathcal{E}_{n \times n}
$$

Indeed, for any $x \in\{-1,1\}^{n}$, the matrix $X:=x x^{t}$ has diagonal entries 1 and satisfies the triangle inequalities; moreover, it is obviously positive semidefinite. Therefore, the metric polytope $\mathrm{MET}_{n \times n}^{ \pm 1}$ and the elliptope $\mathcal{E}_{n \times n}$ are relaxations of the cut polytope $\mathrm{CUT}_{n \times n}^{ \pm 1}$. 


\subsection{The unconstrained quadratic $(0,1)$-programming problem}

The unconstrained quadratic $(0,1)$-programming problem reads

$$
\begin{array}{ll}
\max & \sum_{1 \leq i \leq j \leq n} w_{i j} d_{i} d_{j} \\
\text { s.t. } & d \in\{0,1\}^{n}
\end{array}
$$

where $W=\left(w_{i j}\right)$ is a symmetric matrix of the cost coefficients. The convex hull of the set of feasible solutions to the problem (3.2), after linearization of the objective function, is the polytope $\mathrm{BQP}_{n \times n}$. The polytope $\mathrm{BQL}_{n \times n}$ is a linear relaxation of $\mathrm{BQP}_{n \times n}$, i.e.,

$$
\mathrm{BQP}_{n \times n} \subseteq \mathrm{BQL}_{n \times n},
$$

while a positive semidefinite relaxation of $\mathrm{BQP}_{n \times n}$ is the set $\mathcal{Q}_{n \times n}$ as we have the inclusion:

$$
\mathrm{BQP}_{n \times n} \subseteq \mathcal{Q}_{n \times n} .
$$

Indeed, if $d \in\{0,1\}^{n}$ and $Y:=d d^{t}$, then $Y-\operatorname{diag}(Y)(\operatorname{diag}(Y))^{t}=0$ is, therefore, trivially positive semidefinite!

One may think of other ways of relaxing the condition: $Y=d d^{t}$ for $d \in\{0,1\}^{n}$. For instance, as $(d+v)(d+v)^{t} \succeq 0$ for all $v \in \Re^{n}, Y$ may be constrained to satisfy

$$
Y+\operatorname{diag}(Y) v^{t}+v \operatorname{diag}(Y)^{t}+v v^{t} \succeq 0, \text { for all } v \in \Re^{n} .
$$

Alternatively, one may require that

$$
\left(\begin{array}{l|l}
1 & d^{t} \\
\hline d & Y
\end{array}\right) \succeq 0, \quad \text { where } d:=\operatorname{diag}(Y) .
$$

In fact, as shows the following lemma, these conditions all define the same semidefinite relaxation. $((i) \Longleftrightarrow(i i)$ is contained in [GLS88] and $(i) \Longleftrightarrow(i i i)$ in [BCCP94a].)

LEMMA 3.3 Let $Y$ be a symmetric $n \times n$ matrix, $d \in \Re^{n}$, and $Z:=\left(\begin{array}{l|l}1 & d^{t} \\ \hline d & Y\end{array}\right)$.

The following assertions are equivalent.

(i) $Y-d d^{t} \succeq 0$.

(ii) $Z \succeq 0$.

(iii) $Y+d v^{t}+v d^{t}+v v^{t} \succeq 0$ for all $v \in \Re^{n}$.

Proof. The proof of $(i) \Longleftrightarrow(i i)$ is based on the following observation. Let $b_{0} \in \Re, b \in \Re^{n}$ and $c:=\left(b_{0}, b\right) \in \Re^{n+1}$. Then,

$$
c^{t} Z c=\left(b_{0}+b^{t} d\right)^{2}+b^{t}\left(Y-d d^{t}\right) b .
$$




\begin{tabular}{|c||r|r|r|r|r|r|}
\hline$n$ & \multicolumn{1}{|c|}{3} & \multicolumn{1}{c|}{4} & \multicolumn{1}{c|}{5} & \multicolumn{1}{c|}{6} & \multicolumn{1}{c|}{7} & \multicolumn{1}{c|}{8} \\
\hline total & 100000 & 100000 & 100000 & 100000 & 100000 & 100000 \\
$\lambda_{\min } \geq 0.001$ & 86694 & 80293 & 73576 & 67505 & 61567 & 55880 \\
in $\mathcal{Q}_{n \times n}$ & 8610 & 1498 & 214 & 20 & 3 & 0 \\
\hline
\end{tabular}

Table 2: Matrices from $\mathcal{R}_{n \times n}$ which are also in $\mathcal{Q}_{n \times n}$

For $(i) \Longleftrightarrow(i i i)$, use the identity:

$$
Y+d v^{t}+v d^{t}+v v^{t}=Y-d d^{t}+(d+v)(d+v)^{t} .
$$

This shows that the convex set $\mathcal{Q}_{n \times n}$ is, in some sense, the best possible semidefinite relaxation of $\mathrm{BQP}_{n \times n}$.

Another possible relaxation of the condition: $Y=d d^{t}$ for $d \in\{0,1\}^{n}$, which may seem most natural at first sight, is by requiring that $Y \succeq 0$ and $Y_{i i} \leq 1$ for $i=1, \ldots, n$. In other words, one may consider the convex set

$$
\mathcal{R}_{n \times n}:=\left\{Y \in \mathrm{SYM}_{n \times n} \mid Y \succeq 0, Y_{i i} \leq 1 \text { for } i=1, \ldots, n\right\} .
$$

Clearly, $\mathcal{Q}_{n \times n} \subseteq \mathcal{R}_{n \times n}$. We investigated experimentally how much smaller $\mathcal{Q}_{n \times n}$ actually is with respect to $\mathcal{R}_{n \times n}$. We used MATLAB to generate an $n \times n$ matrix $C$ with entries drawn uniformly from $[-1,1]$. We then scaled the columns $c_{i}$ of $C$ to have norm $l_{i}$, where $l_{i}$ was chosen at random from the unit interval. Then $R:=C^{t} C$ is in $\mathcal{R}_{n \times n}$. Note that, if $R$ is 'close' to the boundary of $\mathcal{R}_{n \times n}$, i.e., if $R$ is near singular, then it is highly unlikely that $R \in \mathcal{Q}_{n \times n}$. We summarize our experiments in Table 2. For each $n=3,4, \ldots, 8$, we generated 100000 matrices $R \in \mathcal{R}_{n \times n}$ in the manner described above. Table 2 indicates how many of them satisfy $\lambda_{\min } \geq 0.001$. The last line of Table 2 indicates the number of matrices that also belonged to $\mathcal{Q}_{n \times n}$. It becomes clear from this simple experiment that optimizing over $\mathcal{Q}_{n \times n}$ instead of $\mathcal{R}_{n \times n}$ should indeed lead to a significant improvement.

\subsection{The maximum stable set problem}

Given a graph $G=(V, E)$ and node weights $c=\left(c_{i}\right)_{i \in V}$, the maximum stable set problem is

$$
\begin{array}{ll}
\max & \sum_{i \in V} c_{i} d_{i} \\
\text { s.t. } & d_{i} d_{j}=0 \text { if } i j \in E \\
& d \in\{0,1\}^{n}
\end{array}
$$

Hence, the stable set polytope $\operatorname{STAB}(G)$ of $G$ is the convex hull of the set of feasible solutions to the program (3.4). The polytope $\operatorname{ODD}(G)$ in $\Re^{n}$ is a relaxation of the 
stable set polytope, i.e.,

$$
\operatorname{STAB}(G) \subseteq \operatorname{ODD}(G) .
$$

When $\operatorname{STAB}(G)=\operatorname{ODD}(G)$, the graph $G$ is said to be $t$-perfect. The set $\operatorname{TH}(G)$ is a positive semidefinite relaxation of the stable set polytope, i.e.,

$$
\operatorname{STAB}(G) \subseteq \operatorname{TH}(G) .
$$

(Indeed, if $d \in\{0,1\}^{n}$ is the incidence vector of a stable set of $G$ and $u:=(1, d) \in$ $\Re^{n+1}$, then the matrix $P:=u u^{t}$ satisfies (2.7); this shows that $d$ belongs to $\mathrm{TH}(G)$.) This definition of $\mathrm{TH}(G)$ is given in [LS91]; other equivalent definitions can be found in [GLS88].

REMARK 3.5 Note that one can optimize in polynomial time over the semidefinite relaxations for the max-cut and stable set problems (as positive semidefiniteness of a matrix can be checked in polynomial time). Let $\mathcal{G}_{c}$ (resp. $\mathcal{G}_{s}$ ) denote the class of the graphs $G$ for which $\mathcal{E}(G)=\mathrm{CUT}^{ \pm 1}(G)$ (resp. $\operatorname{TH}(G)=\operatorname{STAB}(G)$ ). Therefore, the max-cut problem (resp. the stable set problem) can be solved in polynomial time over the class $\mathcal{G}_{c}$ (resp. $\mathcal{G}_{s}$ ). It has been shown that $\mathcal{G}_{s}$ consists of the perfect graphs (see [GLS88]). As a consequence, the stable set problem is polynomial for perfect graphs; this is a nontrivial result for which no other direct proof is known. On the other hand, it is shown in [L94] that $\mathcal{G}_{c}$ consists only of the forests. So, this gives only the easy result that the max-cut problem is polynomial for forests.

\section{Connections}

The purpose of this section is to show the connection existing between the positive semidefinite relaxations of the max-cut problem and of the mximum stable set problem. We recall in Section 4.1 the isomorphism $\varphi$ which provides the correspondence between the polytopes associated with the max-cut problem and the unconstrained $(0,1)$-quadratic problem, as well as their linear and semidefinite relaxations. Section 4.2 makes the link with the stable set problem.

In what follows we shall always suppose that a matrix in $\mathrm{SYM}_{(n+1) \times(n+1)}$ has its entries indexed by the pairs $(i, j)$ for $i, j \in\{0,1, \ldots, n\}$. Given a graph $G$, the graph $G^{\nabla}$ is defined by adding one new vertex adjacent to all original vertices of $G$.

\subsection{Connection between the elliptope $\mathcal{E}\left(G^{\nabla}\right)$ and $\mathcal{Q}(G)$}

Let us consider the linear mapping

$$
\begin{array}{rlll}
\varphi: & \operatorname{SYM}_{(n+1) \times(n+1)} & \longrightarrow & \mathrm{SYM}_{n \times n} \\
X=\left(X_{i j}\right)_{0 \leq i, j \leq n} & \mapsto & Y=\left(Y_{i j}\right)_{1 \leq i, j \leq n}
\end{array}
$$


defined by:

$$
\begin{cases}Y_{i i}:=\frac{1-X_{0 i}}{2} & \text { for all } i=1, \ldots, n, \\ Y_{i j}:=\frac{1+X_{i j}-X_{0 i}-X_{0 j}}{4} & \text { for all } 1 \leq i \neq j \leq n .\end{cases}
$$

The mapping $\varphi$ is many-to-one as the diagonal entries of $X$ do not intervene in the definition of the image $Y=\varphi(X)$. However, an inverse $\varphi^{-1}$ can be defined by requiring that the diagonal entries of $X$ be equal to 1 ; namely,

$$
\begin{array}{rlll}
\varphi^{-1}: & \operatorname{SYM}_{n \times n} & \longrightarrow & \operatorname{SYM}_{(n+1) \times(n+1)} \\
& Y=\left(Y_{i j}\right)_{1 \leq i, j \leq n} & \mapsto & X=\left(X_{i j}\right)_{0 \leq i, j \leq n}
\end{array}
$$

is defined by:

$$
\begin{cases}X_{i i}:=1 & \\ X_{0 i}:=1-2 Y_{i i} & \text { for all } i=1, \ldots, n, \\ X_{i j}:=1+4 Y_{i j}-2 Y_{i i}-2 Y_{j j} & \text { for all } 1 \leq i \neq j \leq n .\end{cases}
$$

As was observed by several authors, the polytopes $\mathrm{CUT}_{(n+1) \times(n+1)}^{ \pm 1}$ and $\mathrm{BQP}_{n \times n}$ are in one-to-one correspondence via the mapping $\varphi$, i.e.,

$$
\varphi\left(\mathrm{CUT}_{(n+1) \times(n+1)}^{ \pm 1}\right)=\mathrm{BQP}_{n \times n}, \quad \varphi^{-1}\left(\mathrm{BQP}_{n \times n}\right)=\mathrm{CUT}_{(n+1) \times(n+1)}^{ \pm 1} .
$$

(This correspondence was observed, in fact, between the cut polytope in 0,1variables and the boolean quadric polytope.) Moreover, the same correspondence holds for the linear relaxations, i.e.,

$$
\varphi\left(\operatorname{MET}_{(n+1) \times(n+1)}^{ \pm 1}\right)=\mathrm{BQL}_{n \times n}, \quad \varphi^{-1}\left(\mathrm{BQL}_{n \times n}\right)=\operatorname{MET}_{(n+1) \times(n+1)}^{ \pm 1} .
$$

Hence, the inequalities of the system (2.3) defining $\mathrm{BQL}_{n \times n}$ correspond to the triangle inequalities. The first two inequalities of the system (2.3) may seem to be more 'natural' than the remaining two ones. They correspond, in fact, to the triangle inequalities through the vertex 0 . Actually, a relaxation of $\mathrm{BQP}_{n \times n}$ using only these two types of inequalities was introduced by Hammer et al. [HHS84], and called a roof dual.

The same correspondence holds also at the level of the semidefinite relaxations.

Proposition $4.1 \varphi\left(\mathcal{E}_{(n+1) \times(n+1)}\right)=\mathcal{Q}_{n \times n}, \quad \varphi^{-1}\left(\mathcal{Q}_{n \times n}\right)=\mathcal{E}_{(n+1) \times(n+1)}$.

Proof. Let $X \in \mathrm{SYM}_{(n+1) \times(n+1)}$ with diagonal entries equal to 1 and $Y=\varphi(X)$. Then, $X \in \mathcal{E}_{(n+1) \times(n+1)}$ if and only if $X \succeq 0$, i.e., $b^{t} X b \geq 0$ for all $b \in \Re^{n+1}$. For $b \in \Re^{n+1}$, set $\sigma:=\sum_{0 \leq i \leq n} b_{i}$. One can check that

$$
b^{t} X b=\sigma^{2}-4 \sigma\left(\sum_{1 \leq i \leq n} b_{i} Y_{i i}\right)+4 \sum_{1 \leq i, j \leq n} b_{i} b_{j} Y_{i j}
$$


Hence, $X \in \mathcal{E}_{(n+1) \times(n+1)}$ if and only if the inequality

$$
\sigma^{2}-4 \sigma\left(\sum_{1 \leq i \leq n} b_{i} Y_{i i}\right)+4 \sum_{1 \leq i, j \leq n} b_{i} b_{j} Y_{i j} \geq 0
$$

holds for all $\sigma \in \Re$ and $\left(b_{1}, \ldots, b_{n}\right) \in \Re^{n}$. At fixed $\left(b_{1}, \ldots, b_{n}\right)$, the inequality (4.2) holds for all $\sigma$ if and only if

$$
\left(\sum_{1 \leq i \leq n} b_{i} Y_{i i}\right)^{2}-\sum_{1 \leq i, j \leq n} b_{i} b_{j} Y_{i j} \leq 0 .
$$

The latter relation holds for all $\left(b_{1}, \ldots, b_{n}\right) \in \Re^{n}$ if and only if the matrix $Y-$ $\operatorname{diag}(Y)(\operatorname{diag}(Y))^{t}$ is positive semidefinite, i.e., if $Y$ belongs to $\mathcal{Q}_{n \times n}$.

There is an analogous correspondence at the level of arbitrary graphs. Namely, let $G=(V, E)$ be a graph with $V=\{1, \ldots, n\}$. Consider the graph $G^{\prime}:=G^{\nabla}$ with node set $V^{\prime}:=V \cup\{0\}$ and edge set $E^{\prime}:=E \cup\{(0, i) \mid i \in V\}$. Let $\varphi_{G}$ denote the one-to-one mapping induced by $\varphi$ between the subspaces $\Re^{E^{\prime}}$ of $\operatorname{SYM}_{(n+1) \times(n+1)}$ and $\Re^{V \cup E}$ of $\mathrm{SYM}_{n \times n}$, i.e.,

$$
\begin{aligned}
& \begin{aligned}
\varphi_{G}: \Re^{\{(0, i) \mid i \in V\} \cup E} & \longrightarrow \Re^{V \cup E} \\
X & \mapsto Y
\end{aligned}
\end{aligned}
$$

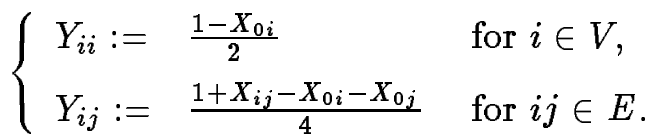

Then it follows that

$$
\begin{gathered}
\varphi_{G}\left(\mathrm{CUT}^{ \pm 1}\left(G^{\nabla}\right)\right)=\mathrm{BQP}(G), \quad \varphi_{G}^{-1}(\mathrm{BQP}(G))=\mathrm{CUT}^{ \pm 1}\left(G^{\nabla}\right) \\
\varphi\left(\mathrm{MET}^{ \pm 1}\left(G^{\nabla}\right)\right)=\mathrm{BQL}(G), \quad \varphi^{-1}(\mathrm{BQL}(G))=\operatorname{MET}^{ \pm 1}\left(G^{\nabla}\right) \\
\left.\varphi_{G}\left(\mathcal{E}\left(G^{\nabla}\right)\right)=\mathcal{Q}(G), \quad \varphi_{G}^{-1}(\mathcal{Q}(G))=\mathcal{E}\left(G^{\nabla}\right)\right) .
\end{gathered}
$$

\subsection{Connection between $\mathcal{Q}(G)$ and $\mathbf{T H}(G)$}

As was already observed (e.g., in [Pad89]), the stable set polytope of a graph $G$ is, in fact, (the projection of) a face of the boolean quadric polytope of $G$; namely,

$$
\operatorname{STAB}(G)=\left\{d \in \Re^{n} \mid\left(d, 0_{E}\right) \in \operatorname{BQP}(G)\right\} .
$$

(Here, $0_{E}$ denotes the all zeros vector of $\Re^{E}$.) This relation extends to the semidefinite relaxations. Namely,

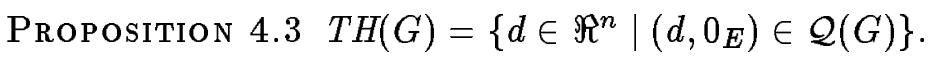


Proof. The proof follows from the definition (2.6) and the equivalence $(i) \Longleftrightarrow(i i)$ of Lemma 3.3.

In other words, the convex body $\operatorname{TH}(G)$ arises from the convex set $\mathcal{Q}(G)$ by intersecting it with the hyperplanes $p_{e}=0$ (for $e \in E$ ) and projecting on $\Re^{n}$. Hence, $\operatorname{TH}(G)$ arises from $\mathcal{Q}(G)$ just in the same way as $\operatorname{STAB}(G)$ arises from $\mathrm{BQP}(G)$. However, $\operatorname{STAB}(G)$ is a face of $\operatorname{BQP}(G)$ (because the inequalities $p_{e} \geq 0$ $(e \in E)$ are valid for $\operatorname{BQP}(G))$; in contrast, $\operatorname{TH}(G)$ is not a face of $\mathcal{Q}(G)$ as the inequalities $p_{e} \geq 0(e \in E)$ are no longer valid for $\mathcal{Q}(G)$.

As a consequence, the body $\operatorname{TH}(G)$ can be expressed directly in terms of the elliptope $\mathcal{E}\left(G^{\nabla}\right)$ as follows: For $d \in \Re^{n}$, define $x \in \Re^{E\left(G^{\nabla}\right)}$ by

$$
\left\{\begin{array}{lll}
x_{0 i}=1-2 d_{i} & & \text { for } i \in V, \\
x_{i j}=1-2 d_{i}-2 d_{j} & & \text { for } i j \in E .
\end{array}\right.
$$

Then,

$$
d \in \mathrm{TH}(G) \Longleftrightarrow x \in \mathcal{E}\left(G^{\nabla}\right) .
$$

In other words, $\operatorname{TH}(G)$ corresponds to the section of the elliptope $\mathcal{E}\left(G^{\nabla}\right)$ by the hyperplanes

$$
x_{i j}=x_{0 i}+x_{0 j}-1
$$

for all $i \in V$.

Finally, let us note that the same connection holds at the level of the linear relaxations; namely,

Proposition 4.5 ODD $(G)=\left\{d \in \Re^{n} \mid\left(d, 0_{E}\right) \in \mathrm{BQL}(G)\right\}$.

Proof. Let $d \in \Re^{n}$ and $x:=\varphi^{-1}\left(d, 0_{E}\right)$ be defined by (4.4). Suppose first that $\left(d, 0_{E}\right) \in \mathrm{BQL}(G)$, i.e., that $x \in \operatorname{MET}^{ \pm 1}\left(G^{\nabla}\right)$. We show that $d \in \operatorname{ODD}(G)$. The relations: $d_{i} \geq 0$ and $d_{i}+d_{j} \leq 1(i j \in E)$ correspond, respectively, to the relations: $x_{0 i} \leq 1$ and $x_{i j} \geq-1$. Let $C$ be an odd cycle in $G$. Then, the inequality $x(C) \geq 2-|C|$ holds; it can be rewritten as $\sum_{i \in V(C)} d_{i} \leq \frac{|C|-1}{2}$. This shows that $d \in \operatorname{ODD}(G)$. Conversely, suppose that $d \in \operatorname{ODD}(G)$; we show that $x \in \operatorname{MET}^{ \pm 1}\left(G^{\nabla}\right)$. For this, we have to check that, if $C$ is a cycle in $G^{\nabla}$ and $F \subseteq C$ with $|F|$ odd, then $x(F)-x(C \backslash F) \geq 2-|C|$ holds. From the above, we can suppose that $C$ is a cycle in $G$ and $F \neq C$. Let $W(F)$ (resp. $W(C \backslash F)$ ) denote the set of nodes of $C$ that are adjacent to two edges in $F$ (resp. in $C \backslash F$ ). Then, the relation $x(F)-x(C \backslash F) \geq 2-|C|$ can be rewritten as

$$
-2 \sum_{i \in W(F)} d_{i}+2 \sum_{i \in W(C \backslash F)} d_{i} \geq 1-|F| .
$$

As $F \neq C, F$ can be decomposed as $F=F_{1} \cup \ldots \cup F_{p}$, where the $F_{h}$ 's are subpaths of $C$. Clearly, the inequality $\sum_{i \in W\left(F_{h}\right)} d_{i} \leq\left\lfloor\frac{\left|F_{h}\right|}{2}\right\rfloor$ (for $h=1, \ldots, p$ ) follows from 
the edge inequalities for $d$. Hence, $\sum_{i \in W(F)} d_{i} \leq \sum_{1 \leq h \leq p}\left\lfloor\frac{\left.\mid F_{h}\right\rfloor}{2}\right\rfloor \leq \frac{|F|-1}{2}$, as $F$ is odd. The inequality (4.6) now follows.

We can summarize the results of the section in the following table, where

$$
\mathcal{L}_{G}:=\left\{X \in \Re^{(n+1) \times(n+1)} \mid X_{i j}=0 \text { for } i j \in E\right\} .
$$

\begin{tabular}{|c|c|c|c|c|}
\hline $\operatorname{STAB}(G)$ & $\longleftrightarrow$ & $\mathrm{BQP}_{n \times n}$ & $\cap$ & $\mathcal{L}_{G}$ \\
\hline $\operatorname{ODD}(G)$ & $\longleftrightarrow$ & $\mathrm{BQL}_{n \times n}$ & $\cap$ & $\mathcal{L}_{G}$ \\
\hline $\mathrm{TH}(G)$ & $\longleftrightarrow$ & $\mathcal{Q}_{n \times n}$ & $\cap$ & $\mathcal{L}_{G}$ \\
\hline
\end{tabular}

\section{Combining linear constraints and semidefinite con- straints}

\subsection{Intersection versus projection}

Quite naturally, a tighter relaxation for each of the problems (3.1) and (3.4) can be obtained by combining the linear relaxation and the semidefinite relaxation. For instance, for the max-cut problem, this amounts to taking the intersection $\operatorname{MET}^{ \pm 1}(G) \cap \mathcal{E}(G)$ of the metric polytope and of the elliptope. In fact, an even better relaxation can be obtained by taking the projection on the edge set only after intersecting the metric polytope and the elliptope. Namely, let $\pi_{E}$ denote the projection of the space $S_{Y M} M_{n \times n}$ on the subspace $\Re^{E}$ indexed by the edge set of $G$. We have the following inclusions:

$$
\operatorname{CUT}^{ \pm 1}(G) \subseteq \pi_{E}\left(\mathcal{E}_{n \times n} \cap \operatorname{MET}_{n \times n}^{ \pm 1}\right) \subseteq \mathcal{E}(G) \cap \operatorname{MET}^{ \pm 1}(G) .
$$

As indicated by the next result, the inclusion

$$
\pi_{E}\left(\mathcal{E}_{n \times n} \cap \operatorname{MET}_{n \times n}^{ \pm 1}\right) \subseteq \mathcal{E}(G) \cap \operatorname{MET}^{ \pm 1}(G)
$$

is, in general, strict.

Proposition 5.3 The inclusion in (5.2) is strict for the graph $G=K_{n} \backslash e, n \geq 7$.

Proof. Set $n=k+3$ where $k \geq 4$ and $a:=\frac{1}{\sqrt{k}}$. Suppose $e=(n-1, n)$ is the missing edge in $G$. Let $x \in \Re^{E}$ be defined by: $x_{i j}=0$ for $1 \leq i<j \leq n-2$, $x_{1, n-1}=a, x_{1, n}=0, x_{n-2, n-1}=0, x_{n-2, n}=a, x_{i, n-1}=x_{i, n}=a$ for $2 \leq i \leq n-3$. Let $X$ denote the symmetric $n \times n$ matrix with diagonal entries 1 and whose off diagonal entries are given by $x$ with $X_{n-1, n}=z$, where $z$ is to be determined. One can check that $X \succeq 0$ if and only if $z=(k-1) a^{2}$, and that $X \in \mathrm{MET}_{n \times n}^{ \pm 1}$ if and only if $2 a-1 \leq z \leq 1-a$. This shows that $x \in \mathcal{E}(G) \cap \operatorname{MET}^{ \pm 1}(G)$. On the other hand, $x \notin \pi_{E}\left(\mathcal{E}_{n \times n} \cap \operatorname{MET}_{n \times n}^{ \pm 1}\right)$ as there is no value of $z$ making $X$ simultaneously positive semidefinite and metric (because $\left.(k-1) a^{2}>1-a\right)$. 
Note that equality holds in (5.2) if $G$ has no $K_{5}$-minor, as $\operatorname{MET}^{ \pm 1}(G)$ coincides then with $\mathrm{CUT}^{ \pm 1}(G)$. Note, however, that $\mathcal{E}(G) \cap \mathrm{MET}^{ \pm 1}(G) \neq \mathrm{CUT}^{ \pm 1}(G)$ for $G=K_{5}$. For this, consider the symmetric $5 \times 5$ matrix $X$ whose diagonal entries are 1 and whose off diagonal entries are equal to $-\frac{1}{4}$. One can easily check that $X \in \mathcal{E}_{5 \times 5} \cap \mathrm{MET}_{5 \times 5}^{ \pm 1} \backslash \mathrm{CUT}_{5 \times 5}^{ \pm 1}$.

Let $\mathcal{G}$ denote the class of graphs for which equality holds in (5.2). The following is known:

THEOREM $5.4 \mathcal{G}$ is closed under taking induced subgraphs and under the clique $k$-sum operation for $k=0,1$.

Proof. We first check that the class $\mathcal{G}$ is closed under taking induced subgraphs. This follows very easily from the following fact. Let $X \in \mathrm{SYM}_{n \times n}$ and set

$$
X^{\prime}:=\left(\begin{array}{cc}
1 & 0 \\
0 & X
\end{array}\right)
$$

Then, $X^{\prime} \in \mathcal{E}_{(n+1) \times(n+1)}$ (resp. $X^{\prime} \in \operatorname{MET}_{(n+1) \times(n+1)}^{ \pm 1}$ ) whenever $X \in \mathcal{E}_{n \times n}$ (resp. $X \in \operatorname{MET}_{n \times n}^{ \pm 1}$ ).

We now show that the clique $k$-sum operation preserves the class $\mathcal{G}$ for $k=0,1$. Let $G_{1}=\left(V_{1}, E_{1}\right)$ and $G_{2}=\left(V_{2}, E_{2}\right)$ be two graphs such that $V_{1} \cap V_{2}$ induces a clique of size $k$ in both $G_{1}$ and $G_{2}$ and there is no edge between a node of $V_{1} \backslash V_{2}$ and a node of $V_{2} \backslash V_{1}$. Then, the graph $G:=\left(V:=V_{1} \cup V_{2}, E:=E_{1} \cup E_{2}\right)$ denotes the clique $k$-sum of $G_{1}$ and $G_{2}$. Set $n_{1}:=\left|V_{1}\right|, n_{2}:=\left|V_{2}\right|$, and $n=|V|=$ $n_{1}+n_{2}-k$. We suppose that $G_{1}$ and $G_{2}$ belong to the class $\mathcal{G}$ and that $k=0,1$. We show that $G \in \mathcal{G}$. For this, let $x \in \mathcal{E}(G) \cap \operatorname{MET}^{ \pm 1}(G)$. We must show that $x \in \pi_{E}\left(\mathcal{E}_{n \times n} \cap \mathrm{MET}_{n \times n}^{ \pm 1}\right)$, i.e., we must find a matrix $X \in \mathcal{E}_{n \times n} \cap \mathrm{MET}_{n \times n}^{ \pm 1}$ such that $x=\pi_{E}(X)$. Let $x_{i}$ denote the restriction of $x$ on the subspace $\Re^{E_{i}}$, for $i=1,2$. Since $G_{i} \in \mathcal{G}$, there exists a matrix $X_{i}^{\prime} \in \mathcal{E}_{n_{i} \times n_{i}} \cap \operatorname{MET}_{n_{i} \times n_{i}}^{ \pm 1}$ so that $x_{i}=\pi_{E_{i}}\left(X_{i}^{\prime}\right), i=1,2$.

In the case $k=0$, we simply take for $X$ the matrix

$$
X=\left(\begin{array}{cc}
X_{1}^{\prime} & 0 \\
0 & X_{2}^{\prime}
\end{array}\right)
$$

one can easily check that $X \in \mathcal{E}_{n \times n} \cap \mathrm{MET}_{n \times n}^{ \pm 1}$.

Suppose now that $k=1$; hence, $n=n_{1}+n_{2}-1$. Let $\{u\}=V_{1} \cap V_{2}$. It is convenient to write the matrices $X_{i}^{\prime}$ (defined above) in the form

$$
X_{1}^{\prime}=\left(\begin{array}{cc}
1 & a^{t} \\
a & X_{1}
\end{array}\right) \quad \text { and } \quad X_{2}^{\prime}=\left(\begin{array}{cc}
1 & b^{t} \\
b & X_{2}
\end{array}\right) \text {, }
$$

where the vertex $u \in V_{1} \cap V_{2}$ corresponds to the first row and column of $X_{1}$ and of $X_{2}$. Set 


$$
X=\left(\begin{array}{ccc}
1 & a^{t} & b^{t} \\
a & X_{1} & a b^{t} \\
b & b a^{t} & X_{2}
\end{array}\right)
$$

We claim that $X \in \mathcal{E}_{n \times n} \cap \operatorname{MET}_{n \times n}^{ \pm 1}$. In order to establish $X \in \mathcal{E}_{n \times n}$, we will use repeatedly the equivalence $(i) \Longleftrightarrow(i i)$ from Lemma 3.3. Since $X_{i}^{\prime} \succeq 0, i=1,2$, we have $X_{1}-a a^{t} \succeq 0$ and $X_{2}-b b^{t} \succeq 0$ and, hence, also

$$
Y:=\left(\begin{array}{cc}
X_{1}-a a^{t} & 0 \\
0 & X_{2}-b b^{t}
\end{array}\right) \succeq 0 .
$$

Using Lemma 3.3 again with $Y$ and $d:=\left(\begin{array}{l}a \\ b\end{array}\right)$, we conclude that $X \succeq 0$. The diagonal entries of $X$ are 1 . Therefore, $X \in \mathcal{E}_{n \times n}$.

It remains to check that $X \in \mathrm{MET}_{n \times n}^{ \pm 1}$, i.e., that $x_{i j}+x_{i k}+x_{j k} \geq-1$ and $x_{i j}-x_{i k}-x_{j k} \geq-1$ for all triples $\{i, j, k\} \subseteq V$. The triangle inequalities involving either $\{i, j, k\} \subseteq V_{1}$ or $\{i, j, k\} \subseteq V_{2}$ are satisfied by our assumption that $G_{1}, G_{2} \in$ $\mathcal{G}$. In particular, we have

$$
\begin{aligned}
& x_{i j}+a_{i}+a_{j} \geq-1 \\
& x_{i j}-a_{i}-a_{j} \geq-1
\end{aligned}
$$

for $i, j \in V_{1} \backslash\{u\}$. There are three possible types of remaining triangle inequalities to consider, denoted below as $(\alpha),(\beta)$ and $(\gamma)$.

Case $(\alpha)$. Consider the triangle inequalities for the triple $\{u, i, j\}$ with $\{u\}=$ $V_{1} \cap V_{2}, i \in V_{1}$ and $j \in V_{2}$. Then, the relations

$$
\begin{gathered}
a_{i}+b_{j}+a_{i} b_{j}+1=\left(a_{i}+1\right)\left(b_{j}+1\right) \geq 0, \\
a_{i}-b_{j}-a_{i} b_{j}+1=\left(a_{i}+1\right)\left(-b_{j}+1\right) \geq 0, \\
-a_{i}-b_{j}+a_{i} b_{j}+1=\left(-a_{i}+1\right)\left(-b_{j}+1\right) \geq 0
\end{gathered}
$$

follow from the fact that $-1 \leq a_{i}, b_{j} \leq 1$.

Case $(\beta)$. Consider the triangle inequalities for the triple $\{i, j, k\}$ with $i, j \in V_{1}$ and $k \in V_{2}$. Then, we have to check the validity of

$$
x_{i j}+b_{k} a_{i}+b_{k} a_{j} \geq-1 \text {. }
$$

We distinguish two subcases according the sign of $a_{i}+a_{j}$. Assume $a_{i}+a_{j} \geq 0$. Then, $\left(a_{i}+a_{j}\right)\left(b_{k}+1\right) \geq 0$ and, hence,

$$
x_{i j}+b_{k}\left(a_{i}+a_{j}\right) \geq x_{i j}-a_{i}-a_{j} \geq-1
$$


using (5.6). Assume $a_{i}+a_{j} \leq 0$. Then $\left(a_{i}+a_{j}\right)\left(b_{k}-1\right) \geq 0$, and hence

$$
x_{i j}+b_{k}\left(a_{i}+a_{j}\right) \geq x_{i j}+a_{i}+a_{j} \geq-1
$$

using (5.5). The other triangle inequalities follow by symmetry.

Case $(\gamma)$. Consider the triangle inequalities for the triple $\{i, j, k\}$ with $i, j \in V_{2}$ and $k \in V_{1}$. The situation is symmetric with case $(\beta)$.

Let us now turn to the maximum stable set problem. In the same way, if $G=(V, E)$ is a graph with $V=\{1, \ldots, n\}$, let $\pi_{V E}$ denote the projection of $\mathrm{SYM}_{n \times n}$ on the subspace $\Re^{V+E}$ (identifying the space of diagonal entries with $\Re^{V}$ ). We have the inclusions:

$$
\begin{aligned}
\left\{\left(d, 0_{E}\right) \mid d \in \operatorname{STAB}(G)\right\} & \subseteq \pi_{V E}\left(\mathrm{BQL}_{n \times n} \cap \mathcal{Q}_{n \times n}\right) \cap \mathcal{H}_{E} \\
& \subseteq \operatorname{BQL}(G) \cap \mathcal{Q}(G) \cap \mathcal{H}_{E} \\
& =\left\{\left(d, 0_{E}\right) \mid d \in \operatorname{TH}(G) \cap \operatorname{ODD}(G)\right\}
\end{aligned}
$$

where $\mathcal{H}_{E}:=\left\{(d, p) \in \Re^{V+E} \mid p_{e}=0\right.$ for $\left.e \in E\right\}$. Note that equality holds throughout (5.7) if $G$ is perfect (as, then, $\mathcal{Q}(G) \cap \mathcal{H}_{E}=\left\{\left(d, 0_{E}\right) \mid d \in \operatorname{STAB}(G)\right\}$ ) or if $G$ is $t$-perfect (as, then, $\left.\operatorname{BQL}(G) \cap \mathcal{H}_{E}=\left\{\left(d, 0_{E}\right) \mid d \in \operatorname{STAB}(G)\right\}\right)$.

REMARK 5.8 An upper bound $\vartheta(G)$ on the stability number $\alpha(G)$ of the graph $G$ is defined by $\vartheta(G):=\max \left\{e^{t} d \mid d \in \mathrm{TH}(G)\right\}$, and called the theta-function of $G$ (see [GLS88]). A. Schrijver ([Sch79]) has shown that $\vartheta(G)$ can be strictly improved by taking $\vartheta^{\prime}(G):=\max \left\{e^{t} d \mid d \in \mathrm{TH}(G), d \geq 0\right\}$. Let us observe that the additional constraint $d \geq 0$ is the first inequality of $(2.5)$, which corresponds to a special case of triangle inequality.

\subsection{Quality of the Approximations}

How large an error can arise when the max-cut is approximated by optimizing over the elliptope or over the metric polytope?

In order to recall some known facts, let us introduce the following notation. Given a graph $G$ and edge weights $w$, let $m c(G, w), \varphi(G, w)$ and $\pi(G, w)$ denote the maximum of $\quad \sum_{1 \leq i<j \leq n} \frac{1}{2} w_{i j}\left(1-X_{i j}\right)$ over $X \in \mathrm{CUT}_{n \times n}^{ \pm 1}, X \in \mathcal{E}_{n \times n}$ and $X \in \operatorname{MET}_{n \times n}^{ \pm 1}$, respectively. In case $w$ is identically 1 on the edges of $G$ (and 0 elsewhere), we write $m c(G), \varphi(G)$ and $\pi(G)$ instead of $m c(G, w), \varphi(G, w)$ and $\pi(G, w)$, respectively.

(a) Relaxation by the elliptope $\mathcal{E}_{n \times n}$. The relaxation over $\mathcal{E}_{n \times n}$ is asymptotically optimal in the following sense. Let $G_{n, p}$ denote a random graph on $n$ vertices with an edge probability $p, 0<p<1$. It has been shown in [DP93] that

$$
\lim _{n \rightarrow \infty} \varphi\left(G_{n, p}\right) / m c\left(G_{n, p}\right) \longrightarrow 1
$$


with probability $1-o(1)$, for any fixed edge probability $p, 0<p<1$. It has been conjectured by Delorme and Poljak that the worst case ratio $\varphi(G) / m c(G)$ is attained for $G=C_{5}$ (the five-cycle) where $\varphi\left(C_{5}\right) / m c\left(C_{5}\right) \doteq 1.131$. The conjecture was 'almost' confirmed by the result of Goemans and Williamson [GW94] who proved $\varphi(G, w) / m c(G, w) \leq 1.138$ for any graph $G$ and any nonnegative edge weights $w$.

(b) Relaxation by the metric polytope $M E T_{n \times n}^{ \pm 1}$. The performance of $\pi(G) / m c(G)$ was studied in [PT92]. In particular, it has been shown that

$$
\lim _{n \rightarrow \infty} \pi\left(G_{n, p_{n}}\right) / m c\left(G_{n, p_{n}}\right) \longrightarrow 2
$$

(with probability $1-o(1)$ ) for certain edge probabilities $p_{n}, p_{n} \rightarrow 0$. This means that the metric approximation $\pi(G)$ can be as bad as possible, since the same worst case ratio is attained by $|E(G)| / m c(G)$. On the other hand, it is well known that $\pi(G, w)=m c(G, w)$ if $G$ is not contractible to $K_{5}$ ([BM86]).

(c) Relaxation by the intersection $\mathcal{E}_{n \times n} \cap M E T_{n \times n}^{ \pm 1}$. It has been proposed in [PR92] to approximate $m c(G, w)$ by

$$
\begin{array}{ll}
\max & \sum_{1 \leq i<j \leq n} \frac{1}{2} w_{i j}\left(1-X_{i j}\right) \\
\text { s.t. } & X \in \mathcal{E}_{n \times n} \cap \mathrm{MET}_{n \times n}^{ \pm 1} .
\end{array}
$$

(It is possible to solve the optimization problem over the intersection in polynomial time using the ellipsoid method as described in [GLS88].)

CONJECTURE 5.9 The worst case of this approximation is attained for the complete graph $K_{5}$, for which the ratio approximation / max-cut is $25 / 24 \doteq 1.04$.

The ratio $25 / 24$ in the conjecture comes from $\varphi\left(K_{5}\right)=25 / 4$ and $m c\left(K_{5}\right)=6$.

Any correlation matrix $X \in \mathcal{E}_{n \times n}$ can be represented by a spherical configuration as follows. Let $X=V^{t} V$ where $V=\left[v_{1}, \ldots, v_{n}\right]$ is a $k \times n$ matrix with columns $v_{i}$. Since $v_{i}^{t} v_{i}=X_{i i}=1$, the vectors $v_{i}$ are unit vectors and, hence, can be considered as points on the unit sphere in $\Re^{k}$. Since $X$ is the Gram matrix of $v_{1}, \ldots, v_{n}$, the vectors are called a Gram representation of $X$. The Gram representation was used by Goemans and Williamson [GW94] to derive the above mentioned result about the worst case bound for positive semidefinite relaxation of the max-cut. If $X$ is a matrix from $\mathcal{E}_{n \times n} \cap \mathrm{MET}_{n \times n}^{ \pm 1}$, its Gram representation has to satisfy some additional constraints.

The following lemma gives a characterization of $X \in \mathcal{E}_{n \times n} \cap \mathrm{MET}_{n \times n}^{ \pm 1}$ in terms of the Gram representation of $X$. In particular it will turn out that in this case the Gram vectors cannot have arbitrary position on the sphere $S_{k}$. This observation may be useful for an error analysis of this tighter relaxation.

We denote by $\cos (a, b)$ the cosine of the angle between the vectors $a$ and $b$. 
Lemma 5.10 Let $X \in \mathcal{E}_{n \times n}$ and its $k \times n$ Gram representation $V$ be given. Let $v_{i}^{t} v_{j}=\cos \alpha_{i j}$, where $0 \leq \alpha_{i j} \leq \pi$. The following two statements are equivalent.

(1) $X \in \mathcal{E}_{n \times n} \cap \mathrm{MET}_{\mathrm{n} \times \mathrm{n}}^{ \pm 1}$.

(2) $\left|\cos \left(v_{k}, v_{i}+v_{j}\right)\right| \leq \cos \left(\frac{\alpha_{i j}}{2}\right)$ for all triples $(i, j, k)$.

Proof. First note that $X \in \mathrm{MET}^{ \pm 1}$ implies that the Gram vectors $v_{k}$ satisfy

$$
\begin{gathered}
v_{i}^{t} v_{j}+v_{i}^{t} v_{k}+v_{j}^{t} v_{k}+1 \geq 0, \\
v_{i}^{t} v_{j}-v_{i}^{t} v_{k}-v_{j}^{t} v_{k}+1 \geq 0
\end{gathered}
$$

for all distinct triples $(i, j, k)$. This is equivalent to

$$
-1-v_{i}^{t} v_{j} \leq v_{k}^{t}\left(v_{i}+v_{j}\right) \leq 1+v_{i}^{t} v_{j}
$$

for all $(i, j, k)$. Note also that

$$
v_{k}^{t}\left(v_{i}+v_{j}\right)=\sqrt{2\left(1+\cos \alpha_{i j}\right)} \cos \left(v_{k}, v_{i}+v_{j}\right) .
$$

Combining the two relations we get

$$
\left|\cos \left(v_{k}, v_{i}+v_{j}\right)\right| \leq \sqrt{\frac{1+\cos \alpha_{i j}}{2}}=\cos \frac{\alpha_{i j}}{2} .
$$

\section{$6 \quad$ Integer Programs in Binary Variables}

We have shown in Section 4.2 that the stable set problem and its relaxation correspond to a constrained max-cut problem. (Another example is the formulation of the graph bisection problems in [PR92].) We shortly address now the question on how to use the previous results for (general) linear and quadratic programs in $(0,1)$ variables. We consider the quadratic $(0,1)$-problem $(\mathrm{QP}-01)$ :

$$
\begin{gathered}
\max x^{t} C x \\
x^{t} A_{i} x \leq b_{i} \quad \text { for } i=1, \ldots, m \\
a_{i}^{t} x \leq \beta_{i} \quad \text { for } i=1, \ldots, k \\
x \in\{0,1\}^{n}
\end{gathered}
$$

and the linear (0,1)-problem (LP-01):

$$
\max c^{t} x \text { subject to (6.2) and (6.3). }
$$

Clearly, (LP-01) is a special case of (QP-01). The problems can be relaxed to the following problem (RELAX): 


$$
\begin{aligned}
& \max \langle C, X\rangle \\
& \left\langle\tilde{A}_{i}, X\right\rangle \leq \tilde{b}_{i} \quad \text { for } i=1, \ldots, N \\
& X \in \mathrm{BQL} \\
& X \in \mathcal{L}_{n \times n} \\
& X \in \mathcal{Q}_{n \times n}
\end{aligned}
$$

Since $x^{t} C x=\operatorname{tr}\left(C x x^{t}\right)=\operatorname{tr} C X$ for $X=x x^{t}$, the problem

$$
\max \langle C, X\rangle \text { subject to } X \in \mathrm{BQL}_{n \times n} \cap \mathcal{Q}_{n \times n}
$$

is a relaxation of $\max x^{t} C x, x \in\{0,1\}^{n}$ (cf. Subsection 3.2). The collection of linear constraints (6.4) is derived from constraints (6.1) and (6.2) using the following steps.

(i) Embedding of linear constraints on the diagonal. Each constraint (6.2) is replaced by

$$
a_{i}^{t} \operatorname{diag}(X) \leq \beta_{i}
$$

since the vertices of $\mathrm{BQP}_{n \times n} \operatorname{satisfy} \operatorname{diag}(X)=x$.

(ii) Linearization of quadratic constraints. Each constraint (6.1) is replaced by

$$
\left\langle A_{i}, X\right\rangle \leq b_{i}
$$

(iii) Generating new quadratic constraints. In [LS91, BCC93] it is proposed to multiply the linear constraints by $x_{j}$ and $1-x_{j}$, to obtain additional quadratic constraints of type (6.1). This produces $2 k n$ new quadratic inequalities:

$$
x_{j}\left(\beta_{i}-a^{t} x\right) \geq 0,\left(1-x_{j}\right)\left(\beta_{i}-a_{i}^{t} x\right) \geq 0
$$

for $i=1, \ldots, k, j=1, \ldots, n$. Finally, [LS91] also suggests to look alternatively at all pairwise products of the constraints

$$
\left(\beta_{i}-a_{i}^{t} x\right)\left(\beta_{j}-a_{j}^{t} x\right) \geq 0,
$$

which yields $k^{2}$ constraints (independent of $n$ ).

In the system (6.7) (or (6.8), respectively) all occurences of $x_{i}$ in a linear term are replaced by $x_{i}^{2}$ for all $i$, and the new quadratic constraints are linearized as described in step (ii). The new system has several remarkable properties (see [LS91]). We can illustrate the effect of the two methods described in (iii) on the following example.

EXAMPle 6.9 Consider the following (LP-01) :

$$
\begin{array}{cl}
\max \sum_{i=1}^{n} x_{i} & \\
x_{i}+x_{j}+x_{k} \leq 2 & \text { for all triples } i, j \text { and } k \text { pairwise different } \\
x_{i} \geq 0 & \\
x_{i} \in\{0,1\} & i=1, \ldots, n
\end{array}
$$


Clearly, the optimum integral solution has value 2 , while the fractional solution (without the last constraint) has the value $2 n / 3$. For $n \geq 4$, the procedure (6.7) leads to the value $4 n / 7$.

The procedure (6.8) leads to the values $8 n / 13,8 n / 14$ and $8 n / 15$ for $n=4$, $n=5$ and $n \geq 6$, respectively. Hence the former method is more efficient for $n=4$, the latter one for $n \geq 6$, and their results coincide for $n=5$.

Finally, adding the positive semidefinite constraint (6.6) to either of (6.7) and (6.8) improves the optimum value to $(n+1) / 4$ for all $n \geq 4$.

REMARK 6.10 The procedure of generating new quadratic constraints and their linearization (described in (ii) and (iii) above) was originaly proposed as a tool to generate new linear constraints to the program (LP-01). However, the results of the previous section 5.1 indicate that it may be advantagous to work in the lifted space (cf. Proposition 5.3).

REMARK 6.11 The constraints of the program (RELAX) consist of two independent parts. While (6.4) depends on the specific constraints of (LP-01) or (QP-01), the constraints (6.5) and (6.6) are always the same, since they express the 01structure of the variables. Since (6.5) and (6.6) themselves are just a relaxation of the max-cut problem, the whole program (RELAX) can be viewed as a relaxation of a 'constrained max-cut problem'. Since any additional known inequalities for the max-cut can be incorporated into our scheme, any progress in solving max-cut is a progress for integer programs.

\section{References}

[BCC93] E. Balas, S. Ceria and G. Cornuejols. A lift-and-project cutting plane algorithm for mixed 0-1 programs. Mathematical Programming, 58:295-324, 1993.

[BCCP94a] E. Balas, S. Ceria, G. Cornuejols and G. Pataki. Polyhedral methods for the maximum clique problem. Technical Report, Carnegie Mellon University, Pittsburgh, USA, 1994.

[BCCP94b] E. Balas, S. Ceria, G. Cornuejols and G. Pataki. Updated semi-definite constraints. Technical Report, Carnegie Mellon University, Pittsburgh, USA, 1994.

[Bar93] F. Barahona. On cuts and matchings in planar graphs. Mathematical Programming, 60:53-68, 1993.

[BM86] F. Barahona and A.R. Mahjoub. On the cut polytope. Mathematical Programming, 36:157-173, 1986 . 
[DP93] C. Delorme and S. Poljak. Laplacian eigenvalues and the max-cut problem, Mathematical Programming 63:557-574, 1993.

[GW94] Michel X. Goemans and David P. Williamson. Improved approximation algorithms for maximum cut and satisfiability problems using semidefinite programming. In Proceedings of the 26th annual symposium on Theory of Computing, Montréal, pages 422-431, 1994.

[GLS88] M. Grötschel, L. Lovász, and A. Schrijver. Geometric algorithms and combinatorial optimization. Springer Verlag, 1988.

[HHS84] P.L. Hammer, P. Hansen and B. Simeone: Roof duality, complementation and persistency in quadratic 0-1 optimization, Mathematical Programming 28 (1984) 121-155.

[HRVW93] C. Helmberg, F. Rendl, R. J. Vanderbei, and H. Wolkowicz. A primal-dual interior point method for the max-min eigenvalue problem. SIAM Journal on Optimization. To appear.

[L94] M. Laurent. The real positive semidefinite completion problem for seriesparallel graphs. Technical Report, 1994.

[LP93] M. Laurent and S. Poljak. On a positive semidefinite relaxation of the cut polytope. Rapport LIENS-93-27, Ecole Normale Supérieure (Paris), 1993. (To appear in Linear Algebra and its Applications.)

[LP94] M. Laurent and S. Poljak. On the facial structure of the set of correlation matrices. Technical report, 1994.

[LS91] L. Lovász and A. Schrijver. Cones of matrices and set-functions and $0-1$ optimization. SIAM Journal of Optimization, 1(2):166-190, 1991.

[Pad89] M. Padberg. The boolean quadric polytope: some characteristics, facets and relatives. Mathematical Programming, 45:139-172, 1989.

[PR92] S. Poljak and F. Rendl. Nonlinear relaxation of the graph-bisection problems, Dimacs Technical Report 92-55, in SIAM Journal on Optimization, to appear.

[PT92] S. Poljak and Zs. Tuza. On the expected relative error of the polyhedral approximation of the max-cut, Report No. 92757-OR (Institut für Diskrete Mathematik, Universität Bonn), Oper. Res. Letters, to appear .

[Sch79] A. Schrijver. A comparison of the Delsarte and Lovász bound. IEEE Transactions on Inf. Th. 25:425-429, 1979. 